\title{
Analyzing Concepts in Action-Frames
}

\author{
Gottfried Vosgerau, Tim Seuchter \& Wiebke Petersen
}

\begin{abstract}
In this paper, we want to argue for a frame-based analysis of action-related concepts as a means for understanding the thesis of grounded cognition, i. e. the thesis that concepts are grounded in sensorimotor processes. We will give an overview of frame analysis and specify frames for action-related concepts. We will then argue for the employment of the frame-based analysis-method and show why this method of analysis has clear advantages over other methods of analysis. The advantages of frames especially become apparent in the capability to specify sensorimotor parts of a concept, as well as in highlighting abstraction mechanisms within concepts. The representation of modal attributes without introducing modal operators is another specific advantage of frames over other formats of representation.
\end{abstract}

\section{Introduction}

The research concerning the problem of grounding language in action so far is mostly empirical. The field is dominated by cognitive psychology and neuroscience, where behavioral and neurological measures are used to relate the possession and processing of conceptual knowledge to sensorimotor processes. The work of Pulvermüller (2005) or Glenberg \& Kaschak (2002), just to name two, clearly showed that there is a connection between concept processing and motorprocesses. However, the theoretical framework of grounded cognition is not yet spelled out in detail, so it remains still an open question of what it exactly means for concepts to be grounded in action.

In this paper we will present and discuss a tool for adequately analyzing concepts. This special kind of concept analysis will then provide a better understanding of what it means for a concept to be grounded in sensorimotor processes. The 
idea is, in short, to analyze action-related concepts within frames. The frame theory in question was originally developed by Barsalou (1992) as a theory of a general format of concept representation; the frame theory underlying this paper is a specified and refined modification of Barsalou's proposal. According to this modification, frames are understood as recursive attribute-value structures. Frames have a lot of advantages over other forms of representing concepts, but due to brevity, we will focus on one special feature of frames concerning action-related concepts: The decomposition of action-related concepts via frame-analysis is able to specify motor-values within these concepts. If motor-values can be analyzed as very basic values in the frame of a certain concept, the conclusion clearly is that this concept is grounded in motor processes. Concepts that do not have motor values as very basic values can hardly be grounded in the relevant sense.

In the following, we will give examples of frames and the idea of special motor values, and we will then show why frames have clear advantages over other formats of representation e. g. first-order predicate logics. First of all, however, we will introduce the strategy of frame analysis.

\section{Concepts and frames}

Barsalou (1992) proposes that the content of concepts (understood as mental entities as opposed to linguistic entities) can be naturally accounted for in terms of frames. Frames as recursive attribute-value structures have been widely used as a general format for knowledge representation, e. g. for capturing linguistic knowledge (Fillmore 1970) or situational knowledge (Minsky 1974). Minsky (1974:1) regards a frame as a "data-structure for representing a stereotyped situation [...]. Once a frame is proposed to represent a situation, a matching process tries to assign values to each frame's terminals, consistent with the markers [restricting the admissible values] at each place." Minsky's aim is to simulate this cognitive process in applications of artificial intelligence. Like Minsky, most approaches consider a frame as a fixed flat set of attributes or slots the values of which get filled by the situation, the linguistic context or whatsoever. By sticking to a fixed set of attributes, those frames essentially reduce to feature lists and categorization to value pattern recognition (1992:23). In contrast, Barsalou (1992:21) argues in favor of frames as "dynamic relational structures whose form is flexible and context dependent". He presents psychological evidence for attribute-value structures de- 
rived from behavioral animal studies. These studies indicate that animals encode stimulus information as attribute values and not as independent features. Furthermore, he gives empirical evidence for the importance of conceptual relations in human cognition. Here we will briefly sketch our frame account which builds on Barsalou's flexible cognitive approach, but provides it with a rigid formal foundation.

The attributes in a concept frame are the general properties or aspects by which the respective concept is described (e. g. SHAPE, LOCATION). ${ }^{1}$ Their values are concrete or underspecified specifications (e. g., [SHAPE: round], [LOCATION: forest]). The attribute values can themselves be complex frames and thus described by additional attributes. For example the value forest of the attribute LOCATION can be further specified by attributes like SIZE or TREE SPECIES. Due to their recursivity, frames are flexible enough to represent information of any desired grade of detail. We assume that attributes in frames assign unique values to objects and thus describe functional relations. Formally, frames can be represented by connected directed graphs where the arcs correspond to attributes. As attributes are functions, no node may have two equally labeled outgoing arcs. The nodes may be labeled by types which restrict the attribute domains and ranges, i. e. the set of objects for which an attribute is adequate and the set of values an attribute can take. Figure 1 shows a simplified frame of the concept 'round shelter in an oak forest'.

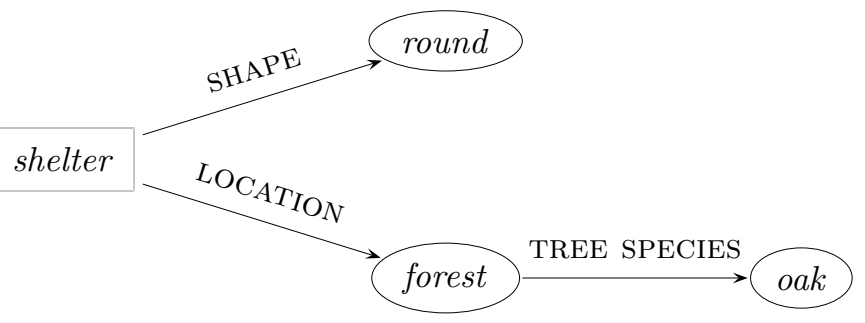

Figure 1: Frame of the concept 'round shelter in an oak forest'

Figure 1 shows two additional notational devices which we use in our graph representations of frames: First, the double border at the shelter node marks it as the central node of the frame; it indicates that the graph represents a frame

1 Throughout this paper

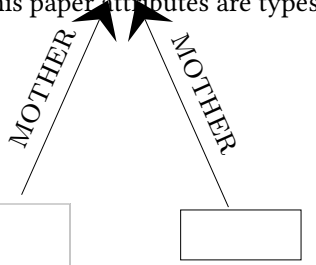


about shelters. Second, by using a rectangular node for the central shelter node, we identify the whole frame as a concept or category frame which corresponds to a 1-place predicate the argument of which is represented by the shelter node. A round central node would indicate instead that it is a frame of a not further specified category member of the category 'round shelter in an oak forest'. As types correspond to 1-place predicates and attributes to functions, the information modeled in the frame can be expressed in the logical formula:

$$
\begin{gathered}
\lambda x . \text { shelter }(x) \wedge \operatorname{round}(\operatorname{SHAPE}(x)) \wedge \text { forest }(\operatorname{LOCATION}(x)) \\
\wedge \text { oak }(\mathrm{TREE} \operatorname{SPECIES}(\operatorname{LOCATION}(x)))
\end{gathered}
$$

In contrast to other frame theories, our frames are capable of representing not only sortal concepts like 'shelter', which denote classical categories, but also relational ones like 'sibling'or 'mother' (Petersen 2007/2015); the referents of the latter concepts are given by a relation to a possessor ('sibling of', 'mother of'). Frames of relational concepts differ from frames of sortal concepts in that they have an additional rectangular node for the possessor argument. Figure 2 shows the frame for the sibling concept. It consists of three nodes, one for the sibling itself (rectangular, double border), one for the person it is the sibling of (rectangular, single border) and one for the mother of both (round). The relation between the two persons is constituted by the fact that they both have the same mother. ${ }^{2}$ This is modeled by the single node to which the two MOTHER-arcs point. Note that in contrast to classical frame accounts our approach does not presuppose that the central node of a frame, i. e. the node which determines what is denoted by a frame, is a root node of the frame graph. ${ }^{3}$

An adequate frame theory needs means of restricting the set of admissible frames. Therefore, frame nodes may be labeled by types. The types are ordered in a type hierarchy which is enriched by appropriateness conditions which constrain the domain and range of attributes. Thus the type signature tells which type of entities can have a certain attribute and of which type the values of each attribute are. Thus we can infer the type of a nodes from the connecting attribute, unless it

2 Note that all frames in this paper are severely simplified. For example, the frame in figure 2 models the sibling concept as being purely determined by the mother relation, leaving aside fathers or socially established family relations.

3 A root of a directed graph is a node from which all other nodes can be reached via paths of directed arcs. 


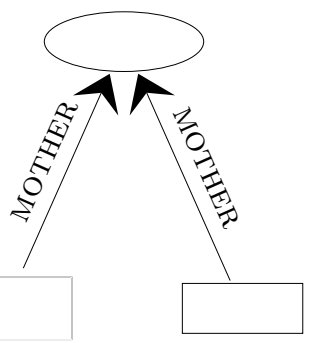

Figure 2: Frame for the concept 'sibling'

is further restricted by other constraints in a particular frame. For example, if a type signature specifies that the domain of the attribute MOTHER is person and that its range is woman, then the information modeled by the frame in figure 2 can be expressed by

$$
\begin{gathered}
\lambda y \lambda x \cdot \operatorname{MOTHER}(x)=\operatorname{MOTHER}(y) \wedge \operatorname{person}(x) \\
\wedge \operatorname{person}(y) \wedge \text { woman }(\operatorname{MOTHER}(x))
\end{gathered}
$$

In contrast to figure 2, figure 1 shows an example of a frame in which the type labels at the nodes effectively restrict the attribute domains and ranges. For instance LOCATION is a very general attribute which applies to all kind of physical objects (not only to shelters) and which takes all kinds of locations as values (not only forests). The formal details of our frame account can be found in Petersen (2007/2015) and Petersen \& Osswald (2014).

Although the informational content of a frame can be expressed in classical logical formulas, it is more natural to assume that concepts are mentally stored in terms of frames than in terms of formulas. As Barsalou (1992) points out, there is empirical evidence for attribute-value sets and relations in cognition. In frames, concepts remain units although they may be highly structured. This unity is concealed in logical formulas by multiple occurring variables. Within frame theory we are not forced to stipulate a fixed arity for each predicate as in predicate logic where predicates are constants. Finally, due to the non-linear structure of frames, one is not required to stipulate an order on the arguments. Rather, substructures can be addressed via labeled symbols instead of ordered argument positions which is cognitively more adequate. By using oscillatory neural networks as a biologically motivated model, Petersen \& Werning (2007) 
give evidence for the cognitive adequacy of our frame model and shows how frames might be implemented in the cortex.

\section{Motor processes in frames}

This framework can now be used to address the question of how the nature of grounded concepts can be specified.

We will introduce some examples of concepts which explicitly involve action and show how action-involvement as a property of a concept can be defined in frames. Because these concepts have a quite simple structure, they serve as an ideal starting point. In the next step, the framework will be generalized to cover other types of (and especially linguistically expressible) concepts.

As we have seen above, frames are characterized by recursive attribute-value structures. Attributes are taken to assign unique values to objects. The values of our interest here are motor-values. They are understood as explicitly representing a movement or a set of movements within a frame. So, the values in these action frames are really parameters that are used in movement control, and not mere numbers or other kinds of abstract values.

Let us start with a simple example: Ants are dynamically representing the location of their nest in terms of the angle to the sun in which the ant has to walk and the number of steps it has to make (Gallistel 1993, Wittlinger et al. 2006). A frame for the ant's representation of the location of the nest is shown in figure 3.

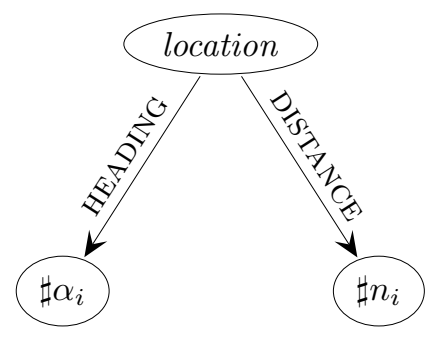

Figure 3: Frame for an ant's representation of the location of the nest

What is crucial here is the meaning of the two values in the lower nodes. Although the graphic shows number variables, the values in the nodes represent 
specific motor-parameters, in this case the angle the ant has to turn and the number of steps it has to make. ${ }^{4}$ As ants are generally held to be unable to count, for ants have no concept of numbers as numbers, those values are simply movements the ant can make. Of course, the ant "knows" how many steps it takes to return to the nest, but it does not "know" it in terms of numbers (Franks et al. 2006). One would be inclined to say that a specific motor-program is running that determines how long and in which direction the ant has to travel to reach the nest. But the ant is not just unable to count, it is also unable to represent these movements as a property of the nest, so we would not want to speak of the ant representing various properties of the nest, namely the heading and the distance (Vosgerau 2007). Rather, it is a good example of a movement representation.

As our interest is in grounded concepts, we need to give an example of a conceptual representation which implies action in the above sense. To stick with the example, a frame thus characterized is shown in figure 4.

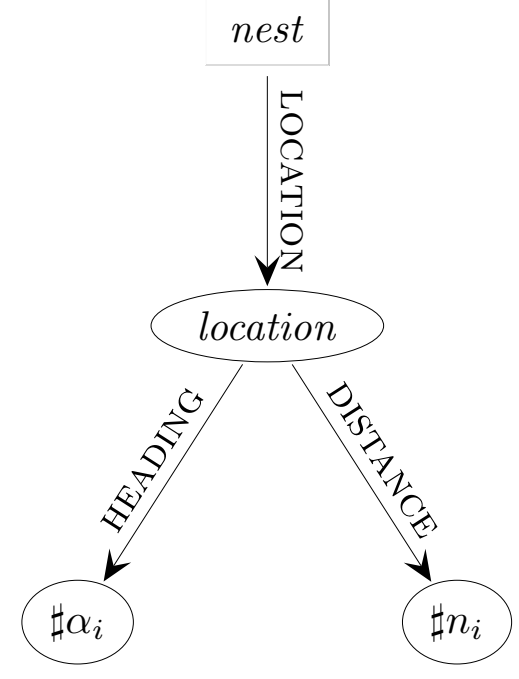

Figure 4: Frame for a conceptual representation of the location of the nest

${ }^{4}$ In the current paper, the dynamic changes of the motor values which occur while actual moving are not in focus. These changes could be modeled as dynamic updates of event frames in a multi-layered architecture as proposed by Naumann (2012). 
Here, the location of the nest is represented as a property of an object, namely the nest. Thus, the representation can in principle be used to represent very different locations of different objects. Technically, this is done by exchanging the specific value of the central node to denote another object. In this sense, this representation can be said to be conceptual: One and the same property can be ascribed to different objects (Newen \& Bartels 2007), and we can clearly analyze one part of the frame to stand for an object (the central node) and another part to stand for a property (the rest of the frame standing for the property of being located at this-and-that point) (Vosgerau 2008).

What we have here is a tool for describing the internal structure of concepts. A concept is constituted by a central node to which properties are assigned by different attributes which assign values. These values, as we have shown, can be motor-processes, in that they represent movements.

The next question obviously has to address the motor-values. What are the constraints to represent motor processes?

Every concept can be analyzed and decomposed. This process of analysis will at one point or another come to a basal level. Basal-level properties are reflected in the end nodes of a concept-frame. One way of dealing with motor processes in concepts is to define them as basal-level-values and to put them in the end nodes of a frame. A concept which contains motor values as basal-level-values can then be said to be grounded in motor abilities. However, if a concept does not contain basal-level-values (i.e. it only contains values that can be further analyzed) or if it contains basal-level-values that are not motoric, then it cannot be characterized as being grounded in motor abilities. (It might still be the case that a concept is grounded in sensory processes if its basal-level-values are sensory values; however, this is not the focus of this paper.)

\section{Significance of frames over other methods of analyzing concepts}

The crucial point so far is that end-values in frames can be motor-parameters. This means that the same values can figure in motor-control mechanisms. This can be displayed most easily when motor processes are represented in frames as well. The possibility of analyzing motor processes (and the specific representations involved in them) in frames directly follows from the claim that frames 


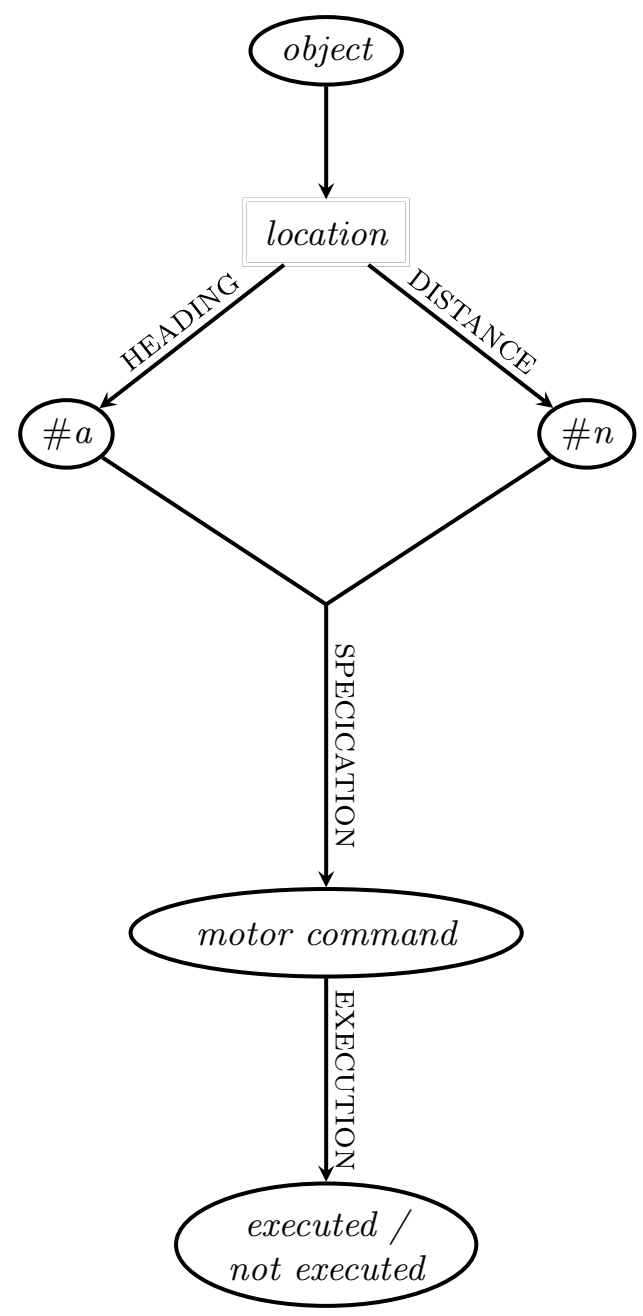

Figure 5: Frame for a specific reaching movement

constitute a general format of representation. In this sense, the frame of a specific reaching movement can be displayed like shown in figure 5 .

According to this (simplified) analysis, the movement is specified by parameters which are in turn further specified and transformed into a specific motor command (cf. diagrams of motor control in Synofzik et al., 2008). This motor 
command can now be executed (i. e. be sent to the muscles) or not. In the latter case, the movement is merely "imagined" or "simulated" (although not necessarily consciously). The important point here is that the upper part of figure 5 has to be understood as a representation of the location of an object whereas the lower part is a representation of a movement; these parts in combination represent a location of an object as a movement (or in terms of a movement).

Usually, motor control mechanisms are depicted in flow-charts (e. g. in Synofzik et al., 2008). The general idea is that each movement (except for reflexes) starts with a "motor intention", which is a usually unconscious and non-conceptual part of motor control (as opposed to the "primary" intention; see Vosgerau \& Synofzik, 2010). For example, if you are going to chop up an onion, your primary intention is to chop up the onion (completely). In contrast, for every cutting of a slice there is a separate motor intention to move the knife in a specific way. This motor intention is usually unconscious and is already in a motor format (something like to move the knife forward while pressing it down). However, the motor intention does not suffice for executing a movement, since several background conditions have to be taken into account. For example, the movement will depend on the actual posture (e.g. where your hand with the knife is) and the surroundings (e. g. where the onion is). Moreover, general facts about the kinematics of the own body have to be taken into account (specified in the "body schema"; see e. g. Vosgerau, 2009). The part of the motor control process, in which the exact parameters of the movement are specified, is often called "specification of movement". The output of the specification is then a motor command which can be sent to the effectors to elicit the movement. However, this last step is not obligatory-indeed, our ability to imagine movements is usually explained by the capacity to run through the motor control sequence without actually executing the movement. Thus, the motor control mechanisms can be used "off-line" to represent a movement rather than to elicit a movement. This aspect of frame analysis provides two main advantages over other formats of description (e. g. logical notations):

First, motor processes can be described as processes in which certain parameters play a role (e. g. motor intentions, posture). Therefore, the specific features of motor control mechanisms can be displayed as shown in figure 6, as opposed to logical notations in which there is no difference (explicitly) displayed between motor-attributes and conceptual attributes (they are, in logical terms, all predicates). 


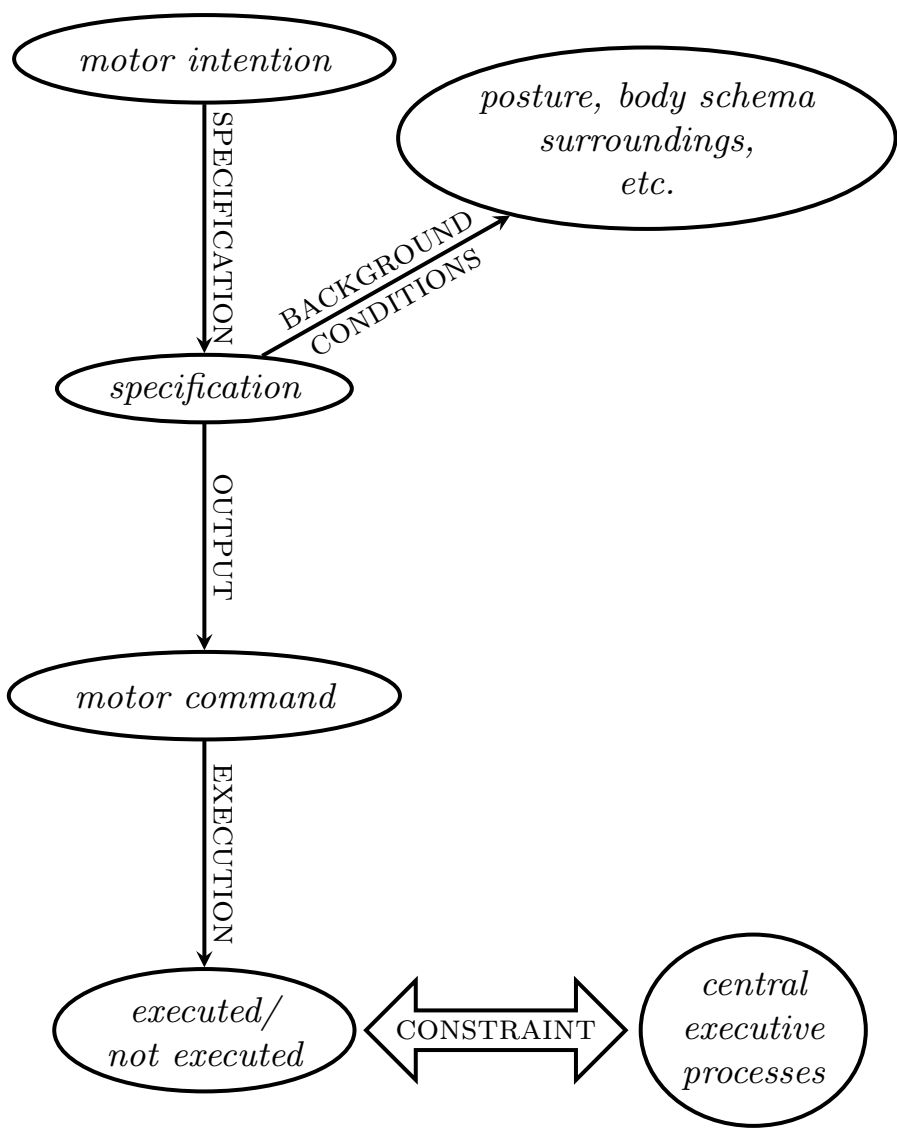

Figure 6: Frame description of motor control

This allows for a reformulation of the question of how grounded abstract concepts are in terms of frames: Are the values of the end-nodes of frames of concepts values that we can also find in sensorimotor processes? In other words: Concept-frames can be combined with motor-control-frames if and only if there is a certain overlap, i. e. if motor-values occur in the concept frame. This formulation of the thesis of grounded cognition directly shows the explanatory advantage of the thesis: It would explain why and how concepts can have a direct impact on our behavior. For example, the motor intentions in the example above (onion 
chopping) could be values that also occur in the concept frame for 'knife', since cutting is an affordance of knifes.

Second, the conceptual categorization of an object does, of course, not automatically lead to a behavior. The movement which is specified by the motorvalues needs not be executed. Thus, entertaining a concept like 'knife' can involve the "activation" of a motor-representation without triggering the according movement (this is what Barsalou Barsalou, 1999 calls "simulation"). In this sense, frames can represent possibilities without introducing a modal operator: The possible movement is represented by motor values which build the basis for a motor command although the motor command is not executed. This fact makes the representation modal in the sense that it represents a merely possible movement and not an actual movement. The specific advantage of frames over other formats of representation is twofold: 1) we can represent modal attributes without introducing modal operators, and 2) there is a straightforward sense in which attribute values can be motor-parameters and not just arbitrary symbols which do not stand in any meaningful relation to motor processing (as it is the case for constants in predicate logic, for example).

\section{Implications for the thesis of grounded cognition}

According to the thesis of grounded cognition, the content of concepts is based on sensorimotor processes, which are constitutive for the relevant concept. What is to be analyzed to understand cognition in general is not mechanisms of computation and abstract symbol manipulation, but rather the relation between conceptualization and basal motor-processes. These motor processes therefore have to be somehow encoded in the concept, they have to be a part or a property of the concept. This view conforms with the fact that these encoded motor processes are not (necessarily) reflected in the verbal structure of the concept. However that holds as well for other functional and physical properties of a concept, which are not reflected in the word form in most cases: The word for the concept 'dog' does not reflect dog-properties nor does it refer to physical or functional properties of dogs (Glenberg \& Kaschak 2002).

Decomposition of concepts via frame analysis is a way to get to a cognitively adequate account of the concept-structure, i. e. the properties assigned to objects by this concept. The most important point, however, is that frame analysis of 
concepts allows us to specifically define what being grounded in action means: If and only if the frame of a concept contains motor-values as base-level-values, it is grounded on motor abilities. Concepts that do not contain such values cannot be said to be grounded in action. Thus, frame analysis goes beyond the rather vague and unspecific claim that concepts are based on modal representations as opposed to abstract symbols (Barsalou 2008). It gives us a clear notion of being grounded which can be applied not only to concepts tout court, but also to parts of concepts. In this way, the "internal" structure of a concept can be taken into account, which leads to a more specific notion of groundedness that can come in well-defined degrees: The transition between grounded and abstract concepts is not a matter of on and off; rather, it is possible that a mid-level concept contains some grounded parts and at the same time already involves other non-grounded, abstract parts.

Weber \& Vosgerau (2012) discuss different ways in which the (unspecific) thesis of grounded action cognition can be interpreted. They argue that three versions can be distinguished: The strong thesis basically collapses into the thesis that thoughts (and a fortiori concepts) are a kind of motor ability. The moderate thesis states that some concepts are constituted by motor processes but others not. The weak thesis amounts to the claim that some motor abilities are necessary to acquire certain concepts but can be lost after acquisition without damage to the concept (i. e. motor abilities are among the acquisition conditions for some concepts but they do not constitute them). In their paper they argue on theoretical and empirical grounds, that the moderate thesis is the most plausible one.

The problem with the strong thesis is present already at a theoretical level, since this thesis faces a serious threat of infinite regress (for details see Vosgerau \& Newen, 2007). Moreover, the thesis is somewhat self-refuting: If it is true that all concepts are a kind of motor ability, then it cannot be said anymore that they are grounded in motor abilities. ${ }^{5}$ Thus, the strong thesis has to be refuted. The weak thesis, on the other hand, can be rejected on the basis of results from empirical studies (see Weber \& Vosgerau, 2012 for details).

Having rejected both the strong and the weak version, there still is evidence that at least some concepts are grounded in motor processes, and this exactly can

5 This is, of course, not a counter-argument against the thesis that thoughts are motor processes. However, this thesis cannot be understood as a thesis of grounded cognition, and this is the important point here. 
be shown in frames. Obviously, not every part of a frame for a concept is based on or related to motor-processes, but at least some parts of it should be interpreted as values for motor-processes. So it is empirical evidence on the one hand that advises for the rejection of both the strong versions of grounded cognition, but on the other hand, conceptual frame-analysis offers good reason to defend and even further refine the moderate version. As reasons for dismissal of a version of a thesis are not sufficient reasons for accepting another version, the contribution of frame-analysis can be seen in the light of giving independent motivations to support the moderate version as described above.

\section{Development of concepts}

The analysis of concepts in terms of frames yields a cognitively adequate representation of concepts. In particular, the sensorimotor grounding of concepts is represented in frames by introducing sensorimotor values. However, concepts apparently differ in degree of groundedness: There are a lot of concepts that are very unlikely to contain motor values (e. g. concepts like 'state', 'freedom', or 'kinship'). Thus, the question of how abstract concepts can develop on the basis of sensorimotor-grounded concepts has to be answered by theories of grounded cognition.

Again, frame analysis turns out to be a useful tool for this task. As shown above, frame analysis is apt to analyze simple representations as they occur in ants. These representations are grounded in the full sense and are not yet abstract enough to be called "concepts". Starting with such simple frames, we have shown that more and more abstract frames of concepts can be developed. First of all, other nodes are added in order to represent a certain property (e. g. the location) of an object as the property of the object. This concept is more abstract since it now allows the representation of one and the same property in different objects and the representation of different properties of the same object (Newen \& Bartels 2007).

One possible next step is to abstract from the concrete values specifying a movement to more abstract forms of motor-representations specifying general schemata of movements. For example, the frame in figure 5 may be appropriate to display my representation of the location of a discussion partner in terms of the head movement I have to execute in order to look at him/her. At the same 
time, it is very plausible that I am able to represent the location of a discussion partner in a more general format that comprises not only head movements but also arm movements (how to reach him/her). Even more abstract are representations in terms of allocentric coordinates that occur both later in the ontogenesis of humans and later in the evolutionary development of species (Vosgerau 2007).

In this way, frame analysis provides a powerful tool to detect abstraction mechanisms that lead from very basic representations that only presuppose sensorimotor abilities to more abstract concepts that get more independent of sensorimotor grounding. Thus, both phylogenetic and ontogenetic development of conceptual abilities is analyzable within frames in terms of different abstraction mechanisms. Of course, it remains an open question how many different abstraction mechanisms there are at work and how far up such mechanisms can get us in terms of abstractness.

\section{Conclusion}

To sum up, we have laid out the foundations of our frame-theory and argued for its advantages over other formats of representing concepts. The frames specified here are capable of representing concepts and information of any desired grade of detail. The frames are not restricted to sortal concepts but can easily represent relational concepts as well. Although it is in principle possible to express the content of the frames in logical formulas, the representation in frames has clear advantages over e.g. predicates of first order logics: no fixed arities have to be stipulated for the predicates, as well as no order of the arguments have to be stipulated, due to the non-linear structure of frames. These advantages concern the formal aspects of frame-representation, so it is worth pointing to one of the psychological incitements for adopting the frame theory: There clearly is evidence that cognitive processes can be understood in terms of attribute-value sets and relations, so the explanatory role of frames within cognitive science is quite clear.

With the frame-theory as a foundation of cognitive representation and processing of concepts, the idea of grounding concepts in action can thus be formulated in a cognitively adequate way and be embedded in a formalizable model of concept-representation. We have shown what it can mean for a concept to be grounded in sensorimotor processes, i. e. to rely on sensorimotor values in frames 
at a very basal level. This notion of groundedness suits well the idea of high-level and low-level processing, where sensorimotor processes are thought to be lowlevel processes which are at play at a very early stage in concept development but do not cease to be a part of higher-level concept processing. Frames reflect this hierarchical understanding of concept development in an appropriate way, as higher-level concepts come along with higher degrees of abstraction within concepts. These degrees and the responsible mechanisms of abstraction can be made visible via frame analysis. The capability of frames to highlight abstraction combined with the possibility to implement modality within representations of motor-based concepts marks frames as a perfect tool for analyzing action-related concepts.

Some further questions remain: As already mentioned above, it is far from clear how concepts exhibiting a very high degree of abstractness could be sensibly understood as grounded on sensorimotor processes. If one adopts our account of analyzing concepts via frames, at least a promising strategy for detecting mechanisms of abstraction is at hand, worth to be persecuted. Another question concerns the scope of the idea of grounded concepts: do literally all concepts have to be grounded in the above sense, or would it already be sufficient for the thesis of grounded cognition that some concepts are straightforwardly grounded, allowing for the possibility of non-grounded concepts? To answer these questions, a lot more empirical and conceptual research on abstract concept grounding has to be done. Our method of analyzing can thus be understood as just a starting point, but a very promising one.

\section{References}

Barsalou, L. W. 1992. Frames, Concepts and Conceptual Fields. In A. Lehrer \& E. F. Kittay (eds.), Frames, Fields and Contrasts, chap. 1, 21-74. Hillsdale, Hove and London: Erlbaum.

Barsalou, L. W. 1999. Perceptual symbol systems. Behavioral and Brain Sciences 22. 577-609. http://journals.cambridge.org/action/displayIssue?jid=BBS\&volume $\mathrm{Id}=22 \&$ issueId $=04 \#$.

Barsalou, L. W. 2008. Grounded Cognition. Annual Review of Psychology 59. 617-645.

Fillmore, C. J. 1970. The Case for Case. In E. Bach \& R. T. Harms (eds.), Universals in Linguistic Theory, 1-88. London, New York, Sydney, Toronto: Holt, Rinehart 


\section{References}

and Winston, Inc.

Franks, N. R., A. Dornhaus, B. G. Metherell, T. R. Nelson, S. A. J. Lanfear \& W. S. Symes. 2006. Not everything that counts can be counted: ants use multiple metrics for a single nest trait. In Proceedings of The Royal Society B, vol. 273, 165-169.

Gallistel, C. 1993. The Organization of Learning. Cambridge MA: The MIT Press.

Glenberg, A. M. \& M. P. Kaschak. 2002. Grounding language in action. Psychonomic Bulletin \& Review 9. 558-565.

Minsky, M. 1974. A framework for representing knowledge. MIT-AI Laboratory Memo 306.

Naumann, R. 2012. Relating ERP-effects to theories of belief update and combining systems. In M. Aloni, V. Kimmelman, F. Roelofsen, G. Sassoon, K. Schulz \& M. Westera (eds.), Logic, Language and Meaning, vol. 7218 Lecture Notes in Computer Science, 160-169. Berlin, Heidelberg: Springer. 10.1007/978-3-642 -31482-7_17. http://dx.doi.org/10.1007/978-3-642-31482-7_17.

Newen, A. \& A. Bartels. 2007. Animal Minds and the Possession of Concepts. Philosophical Psychology 20. 283-308.

Petersen, W. \& M. Werning. 2007. Conceptual Fingerprints: Lexical Decomposition by Means of Frames. In U. Priss, S. Polovina \& R. Hill (eds.), Proceedings of the ICCS 2007, LNAI 4604, 415-428. Berlin: Springer.

Petersen, W. \& T. Osswald. 2014. Concept Composition in Frames: Focusing on Genitive Constructions. In T. Gamerschlag, D. Gerland, R. Osswald \& W. Petersen (eds.), Frames and Concept Types. Applications in Language, Cognition, and Philosophy, vol. 94 Studies in Linguistics and Philosophy, 243-266. Heidelberg, New York, Dordrecht, London: Springer.

Petersen, W. 2015. Representation of Concepts as Frames. In Gamerschlag T., D. Gerland, R. Osswald \& W. Petersen (eds.) Meaning, Frames, and Conceptual Representation, 39-63. Studies in Language and Cognition 2. Düsseldorf: Düsseldorf University Press. (Reprinted from J. Skilters; Toccafondi, F. \& Stemberger, G. (eds.): Complex Cognition and Qualitative Science. Volume 2 of The Baltic International Yearbook of Cognition, Logic and Communication, 151170, University of Latvia, Riga, 2007.)

Pulvermüller, F. 2005. Brain mechanisms linking language and action. Nature Reviews Neuroscience 6. 576-582. 
Synofzik, M., G. Vosgerau \& A. Newen. 2008. Beyond the comparator model: A multifactorial two-step account of agency. Consciousness and Cognition 17. 219-239.

Synofzik, M., G. Vosgerau \& A. Newen. 2008. I move, therefore I am: A new theoretical framework to investigate agency and ownership. Consciousness and Cognition 17. 411-424.

Vosgerau, G. \& A. Newen. 2007. Thoughts, Motor Actions, and the Self. Mind \& Language 22 (1). 22-43.

Vosgerau, G. 2007. Conceptuality in Spatial Representation. Philosophical Psychology 20. 349-365.

Vosgerau, G. 2008. Adäquatheit und Arten mentaler Repräsentationen. Facta Philosophica 10. 67-82.

Vosgerau, G. 2009. Mental Representation and Self-Consciousness. From Basic SelfRepresentation to Self-Related Cognition. Paderborn: mentis.

Vosgerau, G. \& M. Synofzik. 2010. A Cognitive Theory of Thoughts. American Philosophical Quarterly 47. 205-222.

Weber, A. \& G. Vosgerau. 2012. Grounding Action Representations. Review of Philosophy and Psychology 3. 53-69.

Wittlinger, M., R. Wehner \& H. Wolf. 2006. The ant Odometer: Stepping on Stilts and Stumps. Science 312. 1965-1967.

\section{Authors}

Gottfried Vosgerau, Tim Seuchter

Department of Philosophy

Heinrich-Heine-University Düsseldorf

Düsseldorf, Germany

\{seuchter,vosgerau\}@phil.hhu.de

Wiebke Petersen

Departement of Linguistics and Information Science

Heinrich-Heine-University Düsseldorf

Düsseldorf, Germany

petersew@phil.hhu.de 